\title{
Inclusion Exclusion Criteria Not Met Domain
}

National Cancer Institute

\section{Source}

National Cancer Institute. Inclusion Exclusion Criteria Not Met Domain. NCI Thesaurus.

Code C61536.

A subject domain utilized for the submission of information encompassing and representing data, vocabulary or records related to the violation of trial inclusion and exclusion criteria of a subject. 